\title{
Application of the photoreflectance technique to the characterization of quantum dot intermediate band materials for solar cells
}

\author{
E. Cánovas , A. Martí , N. López , E. Antolín , P.G. Linares , C.D. Farmer , \\ C.R. Stanley , A. Luque \\ Instituto de Energia Solar-UPM, ETSIT de Madrid, Ciudad Universitaria sn, 28040 Madrid, Spain \\ Department of Electronics and Electrical Engineering, University of Glasgow, Glasgow G12 8QQ, UK
}

\begin{abstract}
Intermediate band materials rely on the creation of a new electronic band within the bandgap of a conventional semiconductor that is isolated from the conduction and valence band by a true zero density of states. Due to the presence of the intermediate band, a solar cell manufactured using these materials is capable of producing additional photocurrent, thanks to the absorption of photons with energy lower than the conventional bandgap. In this respect, the characterization of these materials by suitable techniques becomes a key element in the development of the new photovoltaic devices called intermediate band solar cells. The technique of photoreflectance is particularly suited to this purpose because it is contact-less and allows the characterization of the material without the need of actually manufacturing a complete device. Using room temperature photoreflectance we have analyzed intermediate band materials based on quantum dots and have been able to identify the energy levels involved. Also, from the photoreflectance data we have demonstrated the overlap of the wave-functions defined by the quantum dots.
\end{abstract}

Keywords: Quantum dots; Intermediate band; Solar cells; Photoreflectance

\section{Introduction}

The intermediate band solar cell (IBSC) is a photovoltaic device conceived to have a limiting efficiency that exceeds that of single gap solar cells due to the exploitation of the properties of intermediate band (IB) materials. This type of material takes its name from the existence of an extra electronic band located between the conduction and valence bands of an ordinary semiconductor (Fig. 1). The basics of IB materials and IBSCs have been described and characterized intensively elsewhere For this reason we will only describe them briefly in the next section, focusing the explanation on the IBSCs based on quantum dots (QDs) nanostructures.

The energy levels of QD nanostructures have been investigated, both theoretically and experimentally However, the most common techniques used for this purpose, such as photoluminescence (PL), PL excitation (PLE), etc. mostly provide information on or are limited to the detection of only the lower energy states of the QD structures, because they are based on emission of light instead of absorption. Other spectroscopic methods like the so-called modulation spectroscopies and particularly its photo-modulated mode, photoreflectance (PR), provide such information since it can detect higher energy transitions in the QDs Furthermore, PR has the advantage of being a contact-less method, and at room temperature yields resolutions achievable by PL only at very low temperature. However, relatively little work has been done on quantum dots at present which exploits the potential of the modulation techniques.

\section{The quantum dot intermediate band solar cell}

A sketch of an IBSC is shown in Fig. 1. Conventional $p$ and $\mathrm{n}$ semiconductor emitters sandwich the IB material. The operating principles of the IBSC predict that, under illumination, the photons reaching the cell are not only able to pump electrons from the valence band (VB) to the conduction band 

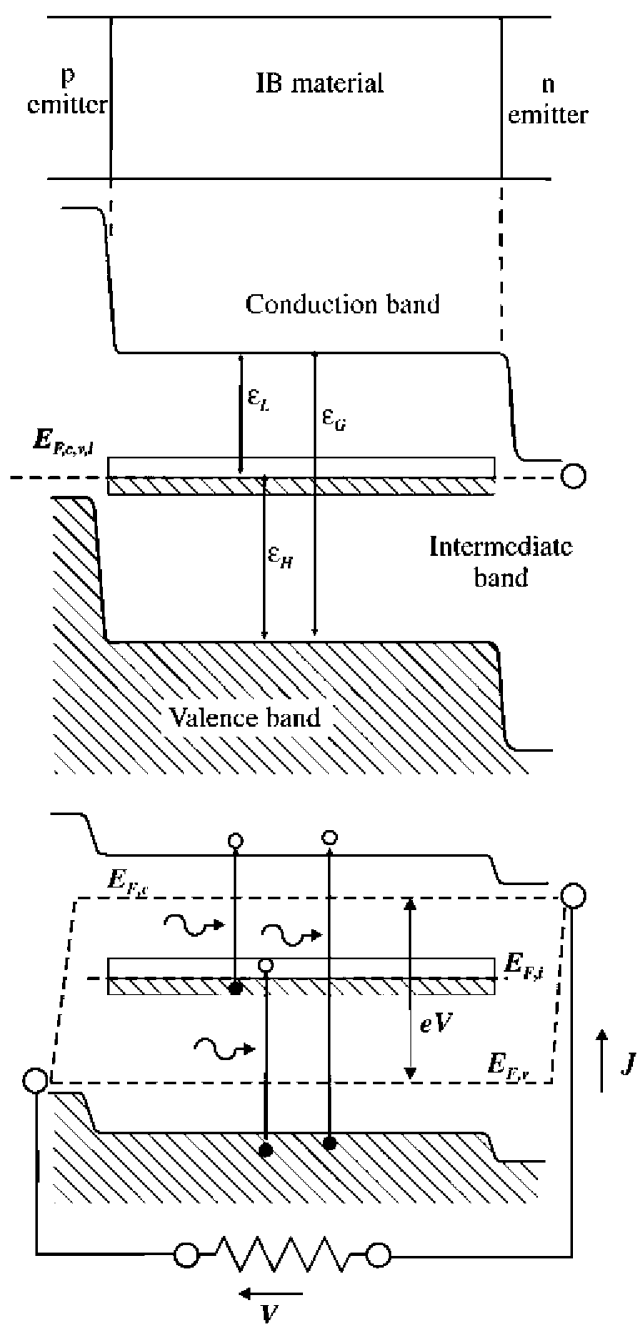

Fig. 1. From top to bottom. Basic structure of an intermediate band solar cell. Simplified bandgap diagram in equilibrium. Simplified bandgap diagram under illumination and forward biased,

(CB), but also from the VB to the IB and from the IB to the $\mathrm{CB}$. In this way, photocurrent is enhanced because photons of energy lower than the bandgap $\varepsilon_{G}$ can contribute to the generation of electrical current in the device. In order to make these absorption processes possible, the IB should be halffilled with electrons. Photocurrent enhancement is not in itself sufficient to improve the efficiency of a solar cell if it is not accompanied by voltage preservation. The output voltage of the IBSC, $V$, is limited by the largest of the bandgaps, being determined by the quasi-Fermi level split between electrons $\left(E_{F, C}\right)$ and holes $\left(E_{F, v}\right)$ as showed in Fig. 1.

The size, shape and composition of QDs dictate the detailed nature of the confining potential for carriers within the bandgap of the host material, and hence they will define the electronic and optical properties of the IB. The IB arises from the confined states that are created by the QDs (Fig. 2). The development of the IBSC using QDs was selected because of the discrete nature of the electron density of states in these nanostructures. Only quantum dots provide a true 0-density of states between their confined states and the CB The quantum well (QW) approach was rejected also due to symmetry selection rules

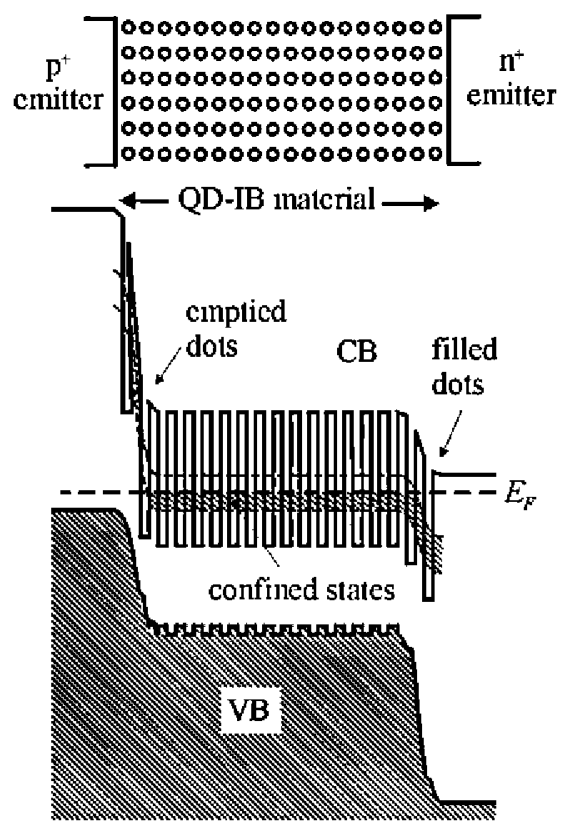

Fig. 2. Illustration of intermediate band formation by means of an array of quantum dots,

The 0-density of states between the confined states and the $\mathrm{CB}$ is also expected to prevent quick relaxation of carriers by means of the so-called phonon-bottleneck effect

\section{Experimental}

IBSCs based on QD prototypes have been manufactured by standard III-V semiconductor processing from stacked layers of self-assembled and self-organised InAs QDs embedded in GaAs barrier material, and grown by molecular beam epitaxy (MBE) in the Stranski-Krastanov growth mode. Their general layer structure is shown in Fig. 3. The sample comprises a stack of 10

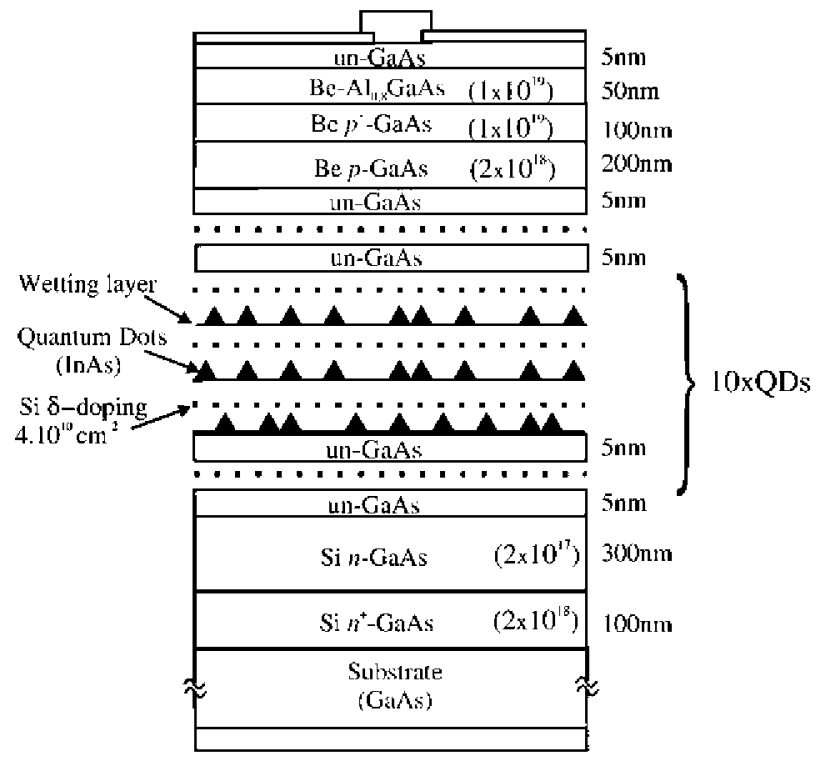

Fig. 3. Layer structure of the quantum dot intermediate band solar cells grown by molecular beam epitaxy. 


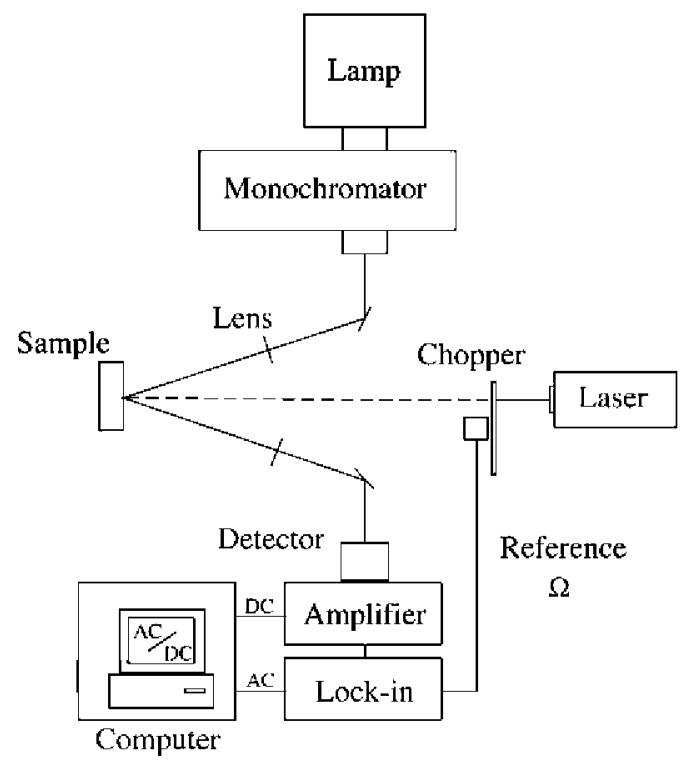

Fig. 4. Photoreflectance setup.

layers of InAs/GaAs QDs with a Si $\delta$-doping layer in between each QD layer to supply electrons to half-fill the IB.

The principal characterisation method of the QD-IBSCs samples described here exploits the potential of PR. Photoreflectance involves the measurement and interpretation of changes in the optical response (reflectivity) of a sample by modulating its electric field . This field is caused by photoexcited electron-hole pairs created by a pump source which is mechanically chopped at a certain frequency $\Omega$, see Fig. 4 . The measurement procedure results in sharp derivative-like spectral features at the wavelengths where inter-band transitions are present.

\section{Results and discussion}

For the development of IBSCs based on QD structures, it is desirable to have an overlap of the wave-functions of the

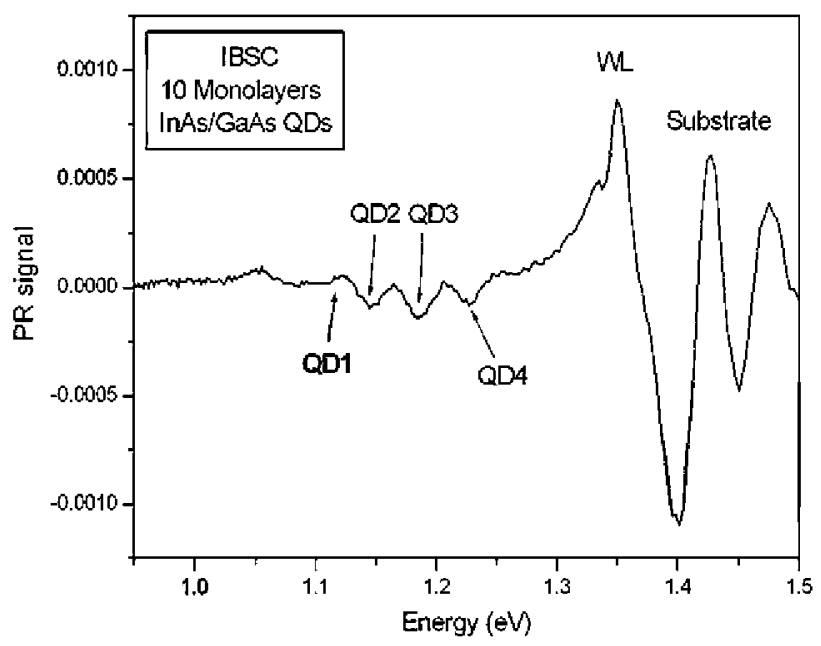

Fig. 5. Photoreflectance of an intermediate band solar cell based on InAs/GaAs quantum dots.

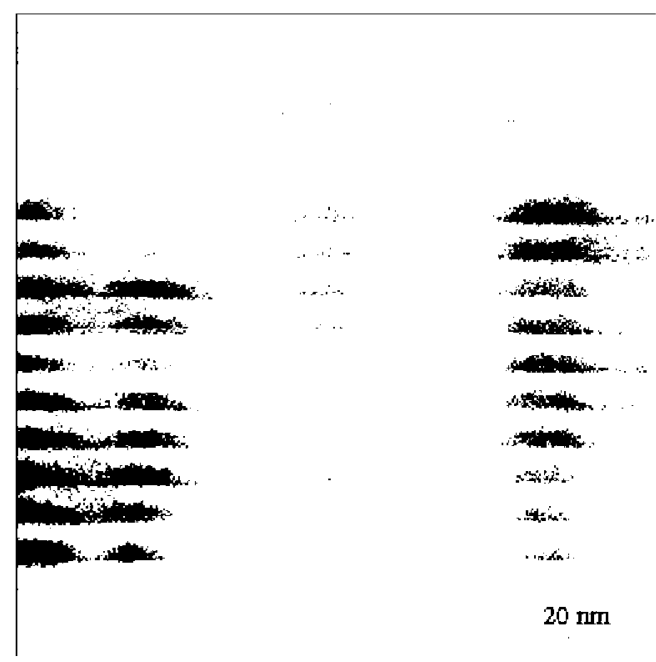

Fig. 6. Transmission electron microscope image of an intermediate band solar cell based on 10 stacks of InAs/GaAs quantum dots.

electrons in the energy levels associated with the QDs, see Fig. 2. In this regard, PR spectroscopy is a very powerful technique to show whether the overlap occurs or not. The derivative-like features of photoreflectance spectra have a particular shape when the carriers are free to move through the crystal which differs from the case when they are in confined states (as they are for single QDs and QWs). The related PR features for confined states have a gaussian-like shape. On the other hand, in the case of confining systems in which there exists an overlap of the wave-functions, they adopt the oscillatory shape that is typical for free carriers. This has been proved for superlattices (SLs) and will be shown and discussed here for QDs.

An easy way for determining the gaussian or oscillatory character of the PR features can be done by comparing the PR plot versus its PR modulus. Gaussian features will maintain the same shape in both, PR and PR modulus, in contrast with those that have an oscillatory behaviour.

Fig. 5 presents the PR spectrum of a typical QD-IBSC with the structure shown in Fig. 3. The four QD related features that are plotted in the Fig. 5 (labelled from QD1 to QD4) have an oscillatory behaviour (in the same way as the GaAs substrate related peak at $1.42 \mathrm{eV}$ ), denoting the free movement of the carriers through the bands generated by the dots. The

Table 1

Experimental PR energy peaks for InAs/GaAs QDs compared with theoretical energies for QDs with a $10 \mathrm{~nm}$ of pyramidal base

\begin{tabular}{llll}
\hline Peak & Type of fitting: & $\begin{array}{l}\text { Experimental energy } \\
(\mathrm{eV})\end{array}$ & $\begin{array}{l}\text { Theoretical energy } \\
(\mathrm{eV})\end{array}$ \\
\hline Substrate & TPM & 1.426 & 1.434, ref.[23] \\
WL2 & Gaussian & 1.349 & 1.388, ref.[23] \\
WL1 & Gaussian & 1.334 & 1.347, ref.[23] \\
QD4 & TPM & 1.227 & 1.225, ref.[4] \\
QD3 & TPM & 1.183 & - \\
QD2 & TPM & 1.147 & - \\
QD1 & TPM & 1.121 & 1.125, ref.[4] \\
defect & Gaussian & 1.053 & 1.03, ref.[22] \\
\hline
\end{tabular}

TPM refers to Aspnes three point method. 


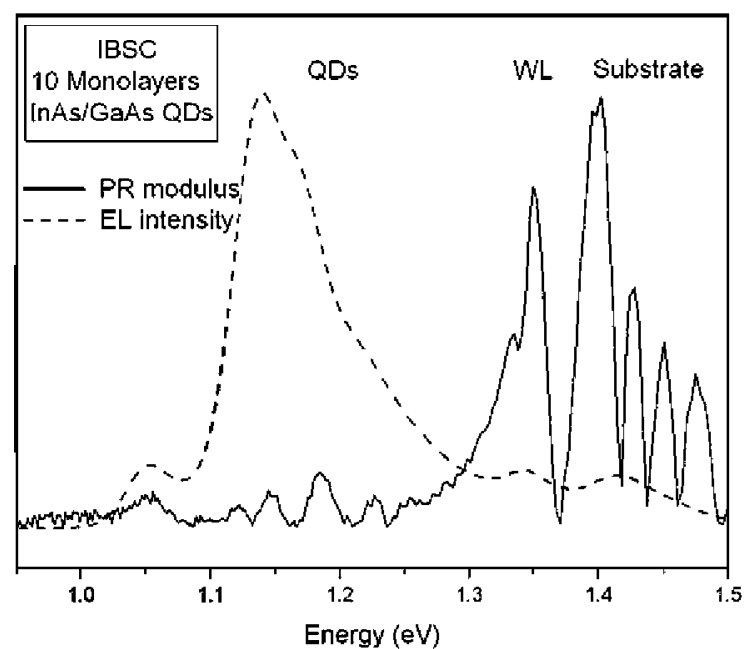

Fig. 7. Comparison between photoreflectance modulus and electroluminescence for an intermediate band solar cell based on InAs/GaAs quantum dots.

lateral and vertical coupling of QDs have been proved in similar samples by low temperature PL measurements but until now, to our knowledge, this is the first time that coupling of QDs nanostructures has been presented and discussed from a qualitative point of view from the shape of features in PR plots.

The peaks due to the presence of the wetting layer have a gaussian-like shape, because of their confined QW nature. The unavoidable presence of a WL below each layer of QDs is a problem in the context of IBSCs because it introduces a continuum density of states from its own energy level up to the CB. However, when the thickness of the WL is small enough, the continuum density of states will be very close to the $\mathrm{CB}$, so the requirements that define the IBSC are once again satisfied, as has been demonstrated recently by an experiment in which the absorption of two sub-bandgap photons mediated by the IB was shown to produce photocurrent in an IBSC

The PR oscillatory features that are present beyond the bandgap of GaAs (at $1.42 \mathrm{eV}$ ) could be related to the so-called Franz-Keldysh oscillations, FKOs. Finally, in Fig. 5, we can see a gaussian-like peak at an energy lower than those associated with the QDs. The confining character of this peak and its energy position, located at $1.053 \mathrm{eV}$, suggests that could be related with the presence of deep-level defects generated during the growth of InAs/GaAs QDs, as has been reported before using deep level transient spectroscopy (DLTS)

From TEM measurements, Fig. 6, that show the high quality and morphology of the nanostructures grown (pyramidal dots with a base length of $\sim 10 \mathrm{~nm}$ ), we have been able to compare our experimental PR data with theoretical estimates. The QD related PR peaks have been fitted by the three-point method (TPM) of Aspnes and the WL related peaks by a gaussian profile. As it is shown in Table 1, the experimental results are in good agreements with the theoretical predictions

Fig. 7 presents room temperature photoreflectance and electroluminescence, EL, measurements of a QD-IBSC. For comparison purposes, the modulus of the PR signal has been taken and the EL peaks have been shifted in energy (to account for the Stokes shift). The EL spectrum was taken from a forwardbiased QD-IBSC made from the same QD wafer, in contrast to the PR spectra which were obtained from unprocessed material. The EL spectrum mainly provides information about the active region of the solar cell. Also, not all of the bands generated by the dots are clearly resolved by the EL technique. The four QD related peaks and the peaks generated by the WL are resolved in a satisfactory way by the PR technique.

As mentioned previously, the distribution, shape and size of the 0-dimensional nanostructures used define the IB in the QD approach to this cell. In general, larger QDs will have a deeper ground state which will translate to a deeper IB However, it implies also the presence of more excited states within the QDs that is an undesirable aspect for the IBSC performance.

\section{Conclusions}

In this paper we have presented for the first time photoreflectance measurements of intermediate band solar cells based on quantum dots. Photoreflectance has shown to be a very powerful method, even at room temperature, for identifying the energy levels of the QD materials compared with techniques based on emission such as electroluminescence. From the photoreflectance data we have demonstrated the overlap of the wave-functions defined by the quantum dots. Also, we have analysed and characterised the different bands generated by the dots, which are in good agreement with theoretical predictions.

\section{Acknowledgments}

This work has been supported by the project FULLSPECTRUM, funded by the European Commission under Contract No. SES6-CT-2003-502620, the Spanish Plan for R\&D Consolider-GENESIS FV (CSD2006-00004), and the Comunidad de Madrid Program NUMANCIA (S-0505/ENE/ 000310). E. Canovas acknowledges the "Plan Nacional de Formación de Personal Investigador" research grant. N.L and E. A acknowledge the UPM financial support through their grant program.

\section{References}

A. Luque, A. Marti, Phys. Rev. Lett. 78 (26) (1997) 5014.

A. Luque, A. Marti, in: A. Martí, A. Luque (Eds.), Next Generation Photovoltaics: High Efficiency through Full Spectrum Utilization, chapter 7 Intermediate-band solar cell, Series in Optics and Optoelectronics, Institute of Physics Publishing, Bristol, 2003.

A. Marti, N. Lopez, E. Antolin, E. Canovas, C. Stanley, C. Farmer, L. Cuadra, A. Luque, Thin Solid Films 511-512 (2006) 638.

M. Grundmann, O. Stier, D. Bimberg, Phys. Rev. Lett. 52 (16) (1995) 11969.

C.E. Pryor, M.E. Pistol, Phys. Rev. B 72 (2005) 205311.

J.Y. Marzin, J.M. Gérard, A. Izraël, D. Barrier, G. Bastard, Phys. Rev. Lett 73 (716) (1994).

G.L. Rowland, T.J.C. Hosea, S. Malik, D. Childs, R. Murray, Appl. Phys Lett. 73 (22) (1998) 3268 .

M. Cardona (Ed.), Modulation spectroscopy, Academic Press, New Cork, 1969. 
J. Misiewicz, P. Sitarek, G. Sek, R. Kudrawiec, Materials Science 21 (3) (2003).

A. Martí, L. Cuadra, A. Luque, IEEE Trans. on Elec. Dev. 48 (10) (2001) 2394.

G.W. Bryant, in: G.W. Bryant, G.S. Solomon (Eds.), Optics of Quantum Dots and Wires, Artech House, Norwood, 2005, Chapter 1.

J.P. Loehr, M.O. Manasreh, Semiconductor Quantum Wells and Superlattices for Long-wavelength Infrared Detectors, Artech House, Boston, 2000.

A.J. Nozik, in: A. Martí, A. Luque (Eds.), Next Generation Photovoltaics: High Efficiency through Full Spectrum Utilization, chapter 9 Quantum Dot Solar Cells, Series in Optics and Optoelectronics, Institute of Physics Publishing, Bristol, 2003.

Y. Nakata, Y. Sugiyama, M. Sugawara, in: M. Sugawara (Ed.), SEMICONDUCTORS AND SEMIMETALS 60, Academic Press, San Diego, 1999, Chap.2.
R.E. Nahory, J.L. Shay, Phys. Rev. Lett. 21 (1968) 1569.

A. Martí, L. Cuadra, A. Luque, IEEE Trans. Electron Dev. 49 (9) (2002) 1632.

D.E. Aspnes, Surface Science 37 (1973) 418.

H. Shen, P. Pranyantal, F.H. Pollak, M. Tomkiewicz, T.J. Drummond, J.N. Schulman, Appl. Phys. Lett. 48 (10) (1986) 563.

G.S. Solomon, J.A. Trezza, A.F. Marshall, J.S. Harris Jr., Phys. Rev. Lett. $76(6)(1996) 952$.

A. Marti, E. Antolin, C.R. Stanley, C.D. Farmer, N. Lopez, P. Diaz, E. Canovas, P.G. Linares, A. Luque, Phys. Rev. Lett. 97 (2006) 247701.

H. Shen, M. Dutta, J. Appl. Phys. 78 (4) (1995) 2151.

M. Kaniewska, O. Engstrom, A. Barcz, M. Pacholak-Cybulska, Mat. Sci. in Semi. Process 9 (2006) 36.

L. Aigouy, T. Holden, F.H. Pollak, N.N. Ledentsov, W.M. Ustinov, P.S.

Kop'ev, D. Bimberg, Appl. Phys. Lett. 70 (25) (1997). 